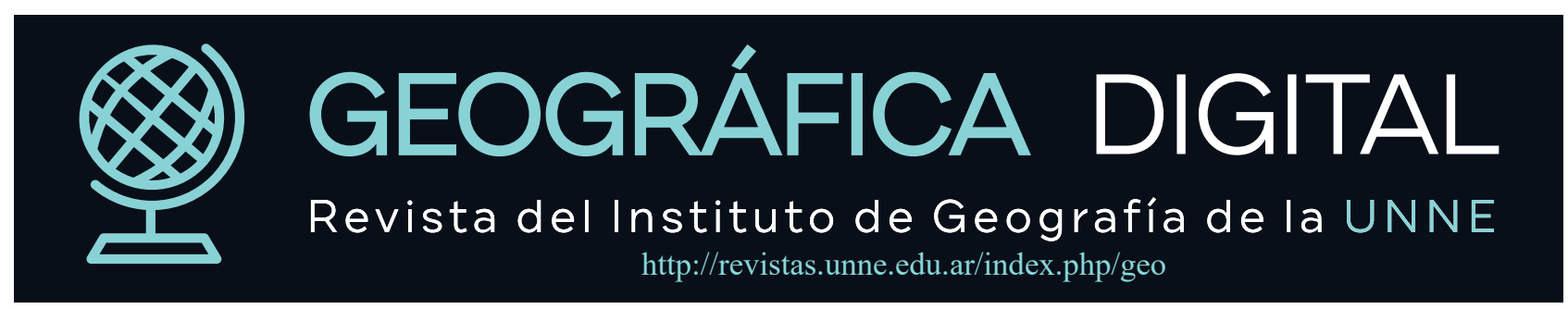

Revista Geográfica Digital, 2019, Vol. 17, N³2, 15-26 pp., DOI: https://dx.doi.org/10.30972/geo.16323584

\title{
Nuevas actividades económicas más allá de las TICs. El surgimiento de Airbnb en Parque Patricios, Ciudad Autónoma de Buenos Aires
}

\author{
New economic activities beyond ICTs. The emergence of Airbnb in Parque \\ Patricios, City of Buenos Aires
}

\author{
Lerena Rongvaux, Natalia ${ }^{1}$ \\ Rodríguez, Luciana ${ }^{2}$ \\ ${ }^{1}$ Instituto de Geografía, Universidad de Buenos Aires. natalia.lerena@filo.uba.ar \\ 22Instituto de Geografía, Universidad de Buenos Aires. arqlucianarodriguez@gmail.com / lucianarodriguez@conicet.gov.ar
}

Palabras Clave:

Airbnb

Renovación urbana

Actividades terciarias

Ciudad de Buenos Aires
Key Words:

Airbnb

Urban Renewal

Service Activities City Of Buenos Aires

\section{R E S U M E N}

Este trabajo aborda el surgimiento de nuevas actividades económicas pertenecientes al sector terciario, que emergen en el marco de procesos de renovación urbana en áreas centrales. En particular, propone analizar la incorporación de ofertas de alquiler temporal a través de la plataforma Airbnb, a la dinámica económica de Parque Patricios, un barrio de la Ciudad Autónoma de Buenos Aires que ha sido objeto de políticas activas de promoción de industrias creativas (TICs) y de mejoras del espacio público, a partir de la creación del Distrito Tecnológico en 2008. La metodología de trabajo se basó en la sistematización exhaustiva y el análisis de los alquileres temporales ofrecidos a través de la plataforma de Airbnb en Parque Patricios, acompañándolo con la oferta de hoteles y restaurantes entre los años 2017 y 2019, lo que permitió detectar su evolución en el corto plazo. Entre los resultados obtenidos, puede observarse el incremento de la presencia de Airbnb en el barrio, al igual que en la nueva oferta gastronómica, como parte de una dinámica de valorización inmobiliaria privada y “espontánea”, pero cuyas condiciones de posibilidad se explican por el papel dinamizador del Estado en el barrio.

\section{A B S T R A C T}

This paper addresses the emergence of new economic activities of the service sector, which develop within the framework of urban renewal processes in central areas. In particular, it proposes to analyze the initiation of temporary rental offers through Airbnb platform, to the economic dynamics of Parque Patricios, a neighborhood of the Autonomous City of Buenos Aires that has been the subject of active policies to promote creative industries (TICs) and public space improvements, accompanying the creation of the Technological District in 2008. The work methodology was based on the exhaustive systematization and the analysis of the temporary rentals offered through the Airbnb platform in Parque Patricios, along with the offer of hotels and restaurants, between years 2017 and 2019, which allowed detecting its evolution in the short term. Among the results obtained, the increase of Airbnb in the neighborhood was verified, as well as the new gastronomic offer, as part of a dynamic of private and "spontaneous" real estate valuation, but whose conditions of possibility are explained by the stimulating role of the State in the neighborhood. 


\section{Renovación urbana y actividades terciarias en la ciudad post-industrial}

Con la consolidación del neoliberalismo como última fase del modo de producción capitalista, las gestiones de las diferentes ciudades han reacondicionado sus herramientas y consolidado un modelo de gestión estatal y planificación urbana, idea que Harvey (1989) tempranamente resumió como el pasaje del gerencialismo al empresarialismo. Green (2005) y De Mattos (2004) lo interpretaron como el paso de la planificación racionalista a la planificación estratégica. Desde este paradigma, la gestión de las ciudades adopta criterios empresariales de eficiencia, competitividad, atractividad, equidad territorial y participación ciudadana, como principios rectores de las políticas urbanas (Fernández Güell, 1997; Borja y Castells, 1997).

En este sentido, la planificación estratégica de las ciudades tiene a la renovación urbana entre uno de sus pilares fundamentales y, la mayoría de las veces, aunque estos procesos de renovación se impulsan desde el Estado, tienen como objetivo final dinamizar el mercado, valorizando el suelo urbano y generando nuevas actividades de consumo orientadas a sectores solventes. Paradigma que se encuadra dentro de un marco económico general, donde los procesos de renovación urbana y las obras de desarrollo de infraestructuras, entre otros, son a la vez nuevas formas de valorización y espacios de colocación del excedente de capital (Harvey, 2013; 2016). Desde este punto de vista, la planificación estratégica busca garantizar el avance del capital sobre áreas anteriormente no rentables, dando lugar a la tensión política entre el mejoramiento a corto plazo de los barrios y su apropiación a mediano plazo por parte del sector privado.

Existen diversas iniciativas por parte de los gobiernos a nivel internacional para renovar áreas degradadas de sus ciudades. Una de las estrategias más difundidas es la patrimonialización de los cascos históricos, generando atractivos que son a la vez singulares de cada ciudad, pero que forman parte de un circuito turístico estandarizado a nivel mundial. En este marco, las operaciones de mejora del espacio público, peatonalización, expulsión del comercio callejero, recuperación de espacios arquitectónicos y promoción de usos culturales y turísticos resultan políticas comunes en la rehabilitación de los centros históricos de diversas metrópolis latinoamericanas desde los años noventa (Vecslir y Rodríguez, 2017). Otras formas de renovación urbana se centran en la generación de atractivos en áreas deprimidas, a partir de la dotación de nuevas infraestructuras y servicios, o bien mediante la creación de hitos urbanos, lo que Borja (2003) señala como monumentalización. También puede mencionarse la generación de polos o distritos económicos, donde se concentran beneficios para la inversión privada, enmarcados en la idea de clúster popularizada por Porter $(1995 ; 1998)$. Dentro de éste paradigma, y tomando como referencia las experiencias del 22@ en el Poblenou de Barcelona, el desarrollo de industrias creativas de alto valor agregado, bajo impacto ambiental y compatibles con el uso del suelo residencial es considerado un instrumento clave del desarrollo territorial. El supuesto que subyace es que la radicación de estas actividades en territorios con una economía urbana degradada genera sinergias locales, que revalorizan económicamente y revitalizan en términos sociales los barrios (Blanco et al., 2015).

En este marco, los cambios en la matriz productiva y en las pautas de localización de las actividades en las ciudades condujeron, a su vez, al creciente desarrollo del sector terciario o de servicios y a la valoración positiva del turismo como nuevo recurso económico para enfrentar la crisis en las ciudades posindustriales (Smith, 1979; Baum, 1994; Britton, 1991). Así, esta actividad comenzó a emplearse como un recurso más para incentivar el crecimiento económico, legitimándose en discursos que apelan a su capacidad de generar empleo, atraer divisas internacionales o disminuir las desigualdades regionales (Almirón et al., 2008). Siguiendo a Harvey (1989), el turismo (una de las estrategias predilectas para las economías urbanas en dificultad) se volvió una actividad propicia para el desarrollo de fragmentos urbanos, proyectos que ejercen atracción social y política, aunque se trate de acciones efímeras frente a problemas urbanos de base o estructurales. Algo que se alinea perfectamente con la idea de proyecto urbano -parcial, fragmentario- que se reivindica desde la planificación estratégica.

Es significativa, durante los últimos años, la emergencia de alojamientos en casas particulares a través de la plataforma Airbnb (AirBed n' Breackfast), una actividad que acompaña al desarrollo del turismo en las ciudades. Asociada a las transformaciones tecnológicas de la era de la información (Borja y Castells, 1997), esta modalidad se enmarca en los sistemas de economía colaborativa, que surgen como alternativa a las formas de mercado tradicional. Se trata de "modelos de negocio en los que se facilitan actividades mediante plataformas colaborativas que crean un mercado abierto para el uso temporal de mercancías o servicios ofrecidos a menudo por particulares" (Comisión Europea, 2016, p.3). Dentro de este sistema, Airbnb es uno de los casos más destacados de economía colaborativa.

Fundada en 2008 como un "mercado comunitario basado en la confianza" (Airbnb, 2017), le permite a las personas alquilar su vivienda de forma temporal, de manera sencilla: basta con ingresar en 
la web y registrar los datos del espacio que se quiere ofrecer -ya sea vivienda entera o habitación privada o compartida-. Su masiva y rápida proliferación (actualmente se desarrolla en 65 mil ciudades del mundo y cuenta con más de 3 millones de anuncios) se debió en gran medida, a la ausencia de una normativa regulatoria en los lugares donde se instala. A su vez, el precio inferior de este tipo de alojamientos en relación a uno tradicional, sumado a los beneficios de hospedarse en una residencia: disponer de una cocina, poder lavar la ropa, la oportunidad de tener una experiencia más "local" interactuando con el anfitrión o los vecinos y, la posibilidad de permanecer en un área "no turística" (dado que estos alojamientos suelen estar más dispersos que los tradicionales) ${ }^{1}$ (Guttentag, 2015), son algunos de los factores que también contribuyeron a su desarrollo.

Esta dinámica puso al descubierto, en muchos casos, importantes problemas de los sistemas de economía colaborativa. En particular, el alza de los precios de los alquileres en los barrios donde se establece y sus efectos de desplazamiento, como ha sido analizado en diferentes ciudades (Yrigoy, 2016). En relación a ello, muchos autores reconocen el estrecho vínculo que existe entre los procesos de renovación urbana y las dinámicas de gentrificación (Hiernaux y González, 2014; Delgadillo, 2015; Janoschka et al., 2014; Sargatal, 2009). El aumento en el precio de los alquileres para los residentes locales debido a una reorientación de las viviendas -al alquiler extranjero y a la alta demanda de los mismos-, el recambio de locales tradicionales por comercios orientados a las nuevas demandas de consumo, o el cambio de ritmo cotidiano en los barrios, son algunos de los rasgos más discutidos que la renovación urbana en las ciudades trae aparejada.

\section{Planificación estratégica en la Ciudad de Buenos Aires. Creación del distrito tecnológico y surgimiento de nuevas actividades económicas}

En la Ciudad Autónoma de Buenos Aires (CABA), están en marcha desde la década del 90 diversas políticas de desarrollo urbano que pueden inscribirse bajo el paradigma de la planificación estratégica (Di Virgilio y Guevara, 2014; Socoloff et al., 2012). En este contexto, se desarrollan una serie de intervenciones estatales específicas que tienen por objetivo lograr un equilibrio territorial entre el norte y centro de la ciudad -áreas atractivas para la inversión y el consumo solvente- y el sur, históricamente relegado por el capital. Las primeras intervenciones urbanísticas en este sector se desarrollaron durante los años 90, e implicaron la patrimonialización del casco histórico, la renovación de Puerto Madero o la puesta en valor del carácter histórico de San Telmo y de La Boca mediante acciones de promoción y embellecimiento urbano. Procesos que atrajeron el interés académico, principalmente por su impacto en la población local y que fueron abordados, en general, desde el enfoque de la sociología y la geografía (Bertoncello y Troncoso, 2014; Ciccolella y Mignaqui, 2009; Gómez Schettini, 2009_a, 2009_b; Herzer, 2008; Rodriguez et al., 2011; Thomasz, 2016).

Sin embargo, a partir del año 2008 nuevas políticas de valorización comienzan a desarrollarse en todas las Comunas del sur de la Ciudad², bajo la implementación de Distritos Económicos. Este es el caso de los barrios de Parque Patricios y Pompeya con la creación de un Distrito Tecnológico (DT) -Ley 2972 y su modificatoria 5234- (Figura 1), así como importantes intervenciones de puesta en valor del espacio público e inversiones en infraestructura de transporte y comunicaciones. Políticas basadas en el supuesto de que, en territorios socioeconómicamente degradados, la radicación de industrias creativas y del conocimiento, de alto valor agregado, bajo impacto ambiental y buena compatibilidad con usos residenciales, genera sinergias locales que impulsan la revalorización de los barrios (Lerena et al., 2016).

El DT retoma la experiencia del 22@ del Poblenou barcelonés, y establece un polígono dentro del cual se incentiva la radicación de empresas de Tecnologías de Información y Comunicación (TICs), mediante la exención de diversos tipos de impuestos (servicios urbanos, residuos secos por construcción, ingresos brutos, sellado por adquisición de inmuebles), durante los primeros 10 años de su establecimiento. Acompañando esta política, en 2015 se inauguró en el centro de Parque Patricios el nuevo edificio de la Jefatura de Gobierno, en consonancia con los principios de monumentalidad y centralidad de la planificación estratégica. Una obra diseñada por el arquitecto Norman Foster, reconocido internacionalmente por su diseño de altos estándares ambientales.

\footnotetext{
$1 \quad$ No obstante, el estudio realizado por Gutiérrez et al. (2017) sobre los patrones de localización de Airbnb en Barcelona sugiere que estos alojamientos se benefician en mayor medida que los hoteles de la proximidad física a los atractivos turísticos de la ciudad, probablemente debido a la facilidad para su expansión en áreas ya consolidadas (Airbnb puede potencialmente expandir su oferta donde ya existen casas y edificios, a diferencia de los hoteles que deben construirse de acuerdo a las pautas de zonificación urbanas). $2 \quad$ Unidades de gestión política y administrativas en las que se divide la CABA desde el año 2005 (Ley Orgánica N 1777).
} 
Figura 1. Mapa de ubicación: Distrito Tecnológico y Parque Patricios
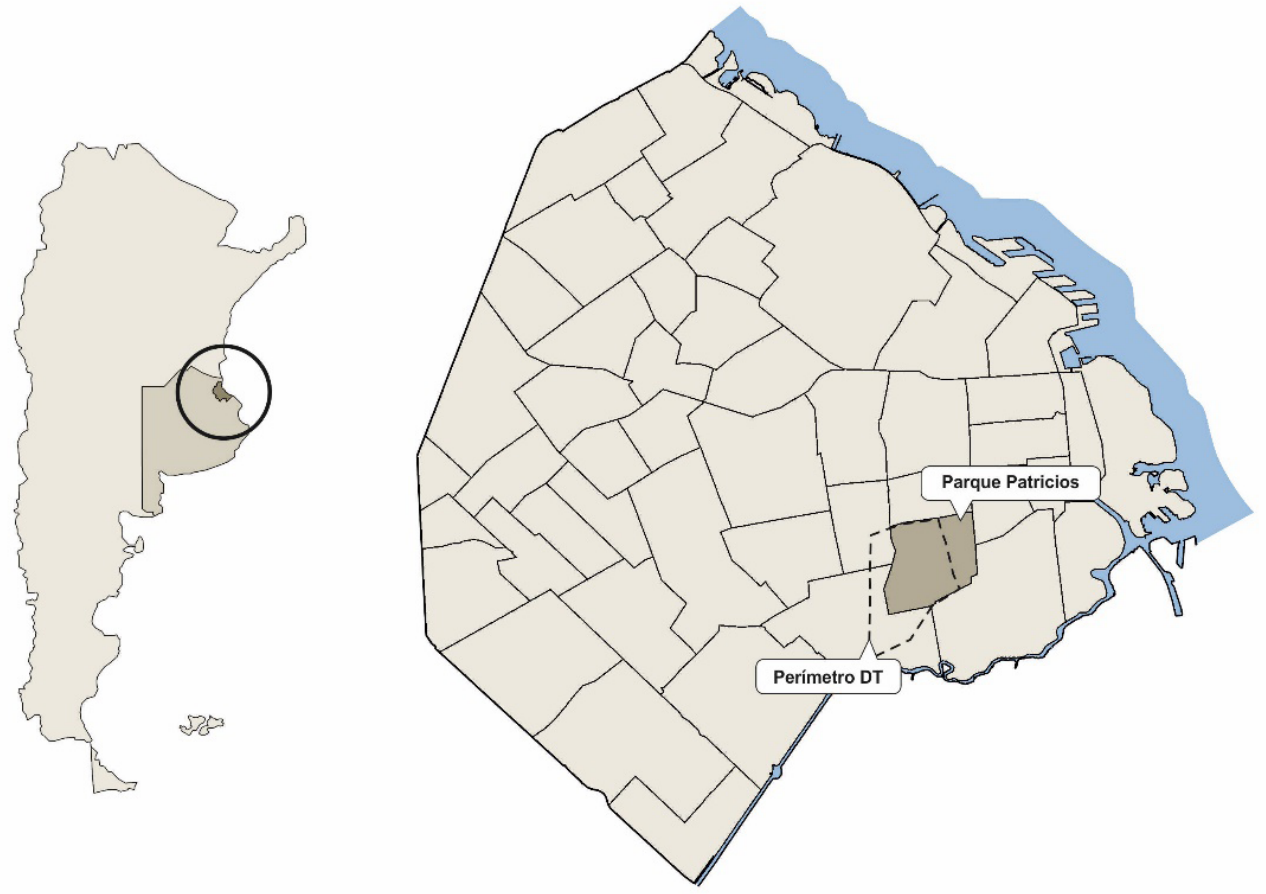

Fuente: Elaboración propia.

Figura 2. Renovación urbana en Parque Patricios

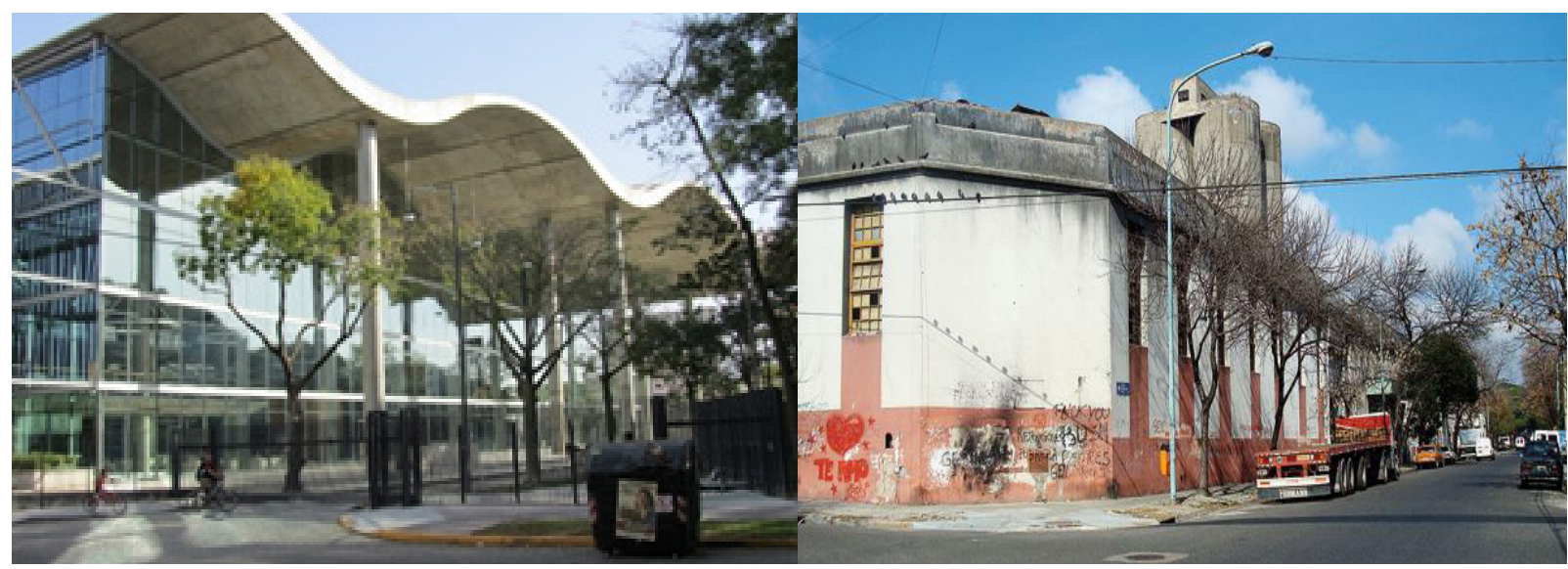

Fuentes: Izq., elaboración propia; Der., Richard Tillman en Lerena et al. 2016.

A su vez, dichas intervenciones han sido apuntaladas por notables transformaciones en el espacio público y en el equipamiento urbano del DT. Entre ellas pueden mencionarse: la rehabilitación del parque principal y otras plazoletas; el mejoramiento de veredas, calles y edificios históricos; aumento de la presencia policial, nueva iluminación y cámaras de seguridad; la instalación de otros edificios públicos, como la sede central de la Policía Metropolitana; importantes obras en materia de telecomunicaciones (instalación de fibra óptica para las empresas y wifi libre en diversos puntos del barrio) e infraestructura en materia de transporte (la construcción de una nueva línea de metro que se conecta con el centro y la zona norte de la ciudad; la creación de un BRT -carriles exclusivos para autobuses--, la construcción de más de $7 \mathrm{~km}$ de nuevas ciclovías y la instalación de dos estaciones de bicicletas públicas).

El conjunto de estas intervenciones ha tenido un significativo impacto en el área del DT, pero sobre todo en el barrio de Parque Patricios, el cual testimonia procesos de valorización relativamente nuevos en la zona sur de la ciudad, muchos de los cuales ya han sido señalados en trabajos anteriores (Socoloff et al., 2012; Lerena, 2016, 2019; Lerena y Rodríguez, 2017; Lerena et al., 2016; Goicoechea, 2017, 2018). Entre los principales, es posible identificar: a) una dinamización del mercado inmobiliario, fundamentalmente a partir del desarrollo de un parque de oficinas orientadas a los usos TICs; b) el surgimiento de una nueva oferta gastronómica en reemplazo de anteriores usos comerciales, vinculada principalmente a los más de 13 mil nuevos trabajadores de las oficinas del DT y la Jefatura; c) la emergencia (desde el 2012 en adelante) de la oferta de alquileres temporales a través de la plataforma Airbnb. 
El análisis de esta última dinámica en particular es objeto del presente trabajo, en tanto no se registran antecedentes de su estudio en la bibliografía local (a excepción de algunas exploraciones realizadas por Lerena y Rodríguez, 2017) y dado que, a partir de los antecedentes internacionales, se reconoce el impacto que podría alcanzar en la dinámica económica del barrio, teniendo en cuenta que un $40 \%$ de sus residentes son inquilinos (INDEC, 2010). En base a lo expuesto, el trabajo se propone responder a los siguientes interrogantes: ¿cómo se está desarrollando la dinámica de Airbnb en Parque Patricios? ¿Dónde se localiza y que relación establece con otras actividades económicas? ¿Qué cualidades presenta este barrio, tradicionalmente fuera del mapa turístico a escala porteña, para que cada vez más personas elijan alojarse temporalmente allí? ¿Qué relación existe entre la emergencia de esta actividad y las políticas de renovación urbana en curso?

\section{Consideraciones metodológicas}

A fin de indagar en el surgimiento y evolución en el alquiler temporal vía Airbnb en Parque Patricios, se definió como estrategia metodológica realizar un relevamiento exhaustivo de la oferta de alojamientos de la plataforma en dos momentos: octubre de 2017 y mayo de 2019. Para ello, se ingresó el nombre "Parque Patricios, Buenos Aires, Argentina" en la web de Airbnb (https://www.airbnb.com.ar/), en las fechas indicadas y sin aplicar filtros de búsqueda -fecha, cantidad de huéspedes, tipo de alojamiento, precio, reservación inmediata u otros filtros-. De esta forma, se registraron todos los alojamientos localizados en el barrio a partir de la información que arrojaba el mapa interactivo, descartando aquellas publicaciones que correspondían a otros barrios (aún publicadas erróneamente como Parque Patricios) y eliminando ofertas duplicadas. También se realizó un relevamiento general de la oferta para todos los barrios de la CABA (ingresando el nombre de cada barrio y registrando el número total de alquileres indicados en la parte inferior de la página), a fin de poder comprar la situación específica de Parque Patricios en relación a la dinámica de Airbnb en la ciudad para 2017 y 2019 (Figura 3).

Los resultados se plasmaron en un mapa con la localización de la oferta en ambos momentos, información que se complementó con la localización de restaurantes y hoteles a partir del relevamiento obtenido en la web TripAdvisor (https://www.tripadvisor.com.mx/) en las mismas fechas (Figuras 4 y 5). Para ello, se ingresó dentro del "Portal de Viajes: Buenos Aires", desplegando el mapa por barrios de "Explora cualquier destino de Buenos Aires", realizando un zoom al sector de Parque Patricios. Esto permitió obtener información complementaria acerca de la dinámica de servicios gastronómicos y hospedaje formal, detectando la existencia de tendencias comunes de crecimiento y localización.

Por otro lado, se ingresó en cada una de las publicaciones de hospedaje vía Airbnb, para identificar el año en el que los anfitriones (personas que ofrecen el alojamiento) se registraron en la plataforma; dato que se tomó como "momento de inicio de la oferta". A su vez, se sistematizaron todos los comentarios que aludían al barrio en cada una de las ofertas publicadas, tanto por parte de los anfitriones como de los visitantes, a partir de cuatro variables cualitativas: a) cualidades del barrio, b) accesibilidad y oferta de transporte público, c) oferta comercial barrial y d) presencia de atractivos turísticos. A su vez, se registró la procedencia de los visitantes, a fin de determinar el perfil del público que hace uso del servicio. Esto se tradujo en una infografía que ordena la información obtenida (Figura 6).

Por último, se registró el precio de los alquileres temporales en dólares para los dos momentos del relevamiento, datos que se compararon con los precios del mercado inmobiliario del barrio, a través de un relevamiento periódico realizado por el Ministerio de Desarrollo Urbano del Gobierno de la Ciudad de Buenos Aires (MDU-GCBA) y de relevamientos propios, a partir de buscadores web especializados (Zona Prop, Argenprop, Buscainmueble e Inmuebles Clarín) entre los años 2001 y 2017. En el caso de los relevamientos oficiales, se controlaron, validaron y eventualmente corrigieron los errores originales de los datos, continuando con trabajos previos (Lerena, 2019). Esto permitió, aunque de manera incipiente, una aproximación al impacto que el surgimiento de Airbnb podría ejercer en relación a la dinámica inmobiliaria de Parque Patricios.

\section{Alquiler temporal: emergencia de una actividad espontánea en el distrito tecnológico}

Las políticas de renovación urbana puestas en marcha para el DT, especialmente en el barrio de Parque Patricios, están centradas en la consolidación de un área de actividad económica especializada: las industrias creativas, en particular las TICs. Sin embargo, en los últimos años puede observarse el surgimiento de nuevas actividades que también han contribuido al nuevo dinamismo de este tradicional barrio porteño, como es el caso de la oferta de alquileres temporales vía Airbnb. No obstante, se considera 
que su desarrollo tiene como condición de posibilidad la dinámica de renovación urbana en curso y, en particular, el papel central del Estado en la recualificación del barrio. Desde la lógica de las políticas implementadas, esta nueva actividad económica podría analizarse como un efecto deseado, aunque no planificado de dicho proceso.

A partir del relevamiento realizado para este trabajo, puede observarse que en Parque Patricios comienzan a ofertarse viviendas y habitaciones de alquiler temporal a través de Airbnb desde enero 2012, momento en el que el barrio ya contaba con nuevos atributos de renovación urbana: subterráneo, mejoramiento del espacio público y espacios verdes, nuevos locales comerciales, entre otros. Desde 2015 la oferta crece, llegando a registrarse 43 propiedades ofertadas a la fecha del segundo relevamiento (mayo de 2019), algunas de las cuales pertenecen al mismo oferente, y orientadas, casi en su totalidad, al ocio y el turismo ${ }^{3}$.

Si se compara la cantidad de unidades ofrecidas en Parque Patricios con los barrios más buscados de la ciudad (los más consolidados en términos turísticos: Palermo, San Telmo, Almagro, Monserrat, San Nicolás, Belgrano, Recoleta, entre otros), se observa que la oferta es aún muy baja, representando en 2019 cerca del $14 \%$ de la cantidad ofrecida en aquellos barrios (43 frente a más de 300 ofertas). No obstante, al comparar la evolución entre los dos años de relevamiento (2017 y 2019), el aumento de las ofertas en Parque Patricios es significativo, pasando de 20 a 43 unidades.

Con respecto a la dinámica general de ofertas, la Figura 3 permite observar la tendencia al crecimiento de ofertas de alquiler temporal vía Airbnb en la ciudad, en términos generales. En particular, es significativa la dinámica de algunos de los barrios "no turísticos", entre los cuales se encuentra Parque Patricios, que pasan de la categoría "1 a 29" a "30 a 99" ofertas, mientras otros (particularmente los más consolidados) permanecen en la misma categoría entre ambos relevamientos.

Como puede observarse en las Figuras 4 y 5, los alquileres en Parque Patricios se localizan mayormente en el cuadrante noroeste del barrio, delimitado por Av. Caseros, y las calles Deán Funes, Av. Garay y Sánchez de Loria. Esto refuerza la centralidad del subsector, tradicionalmente el de mejor calidad urbana y el más consolidado, también en relación a la radicación de empresas TIC a partir de la Ley de Distrito Tecnológico (Goicoechea, 2016).

Figura 3. Comparación de la oferta de Airbnb en la Ciudad Autónoma de Buenos Aires, 2017-2019.
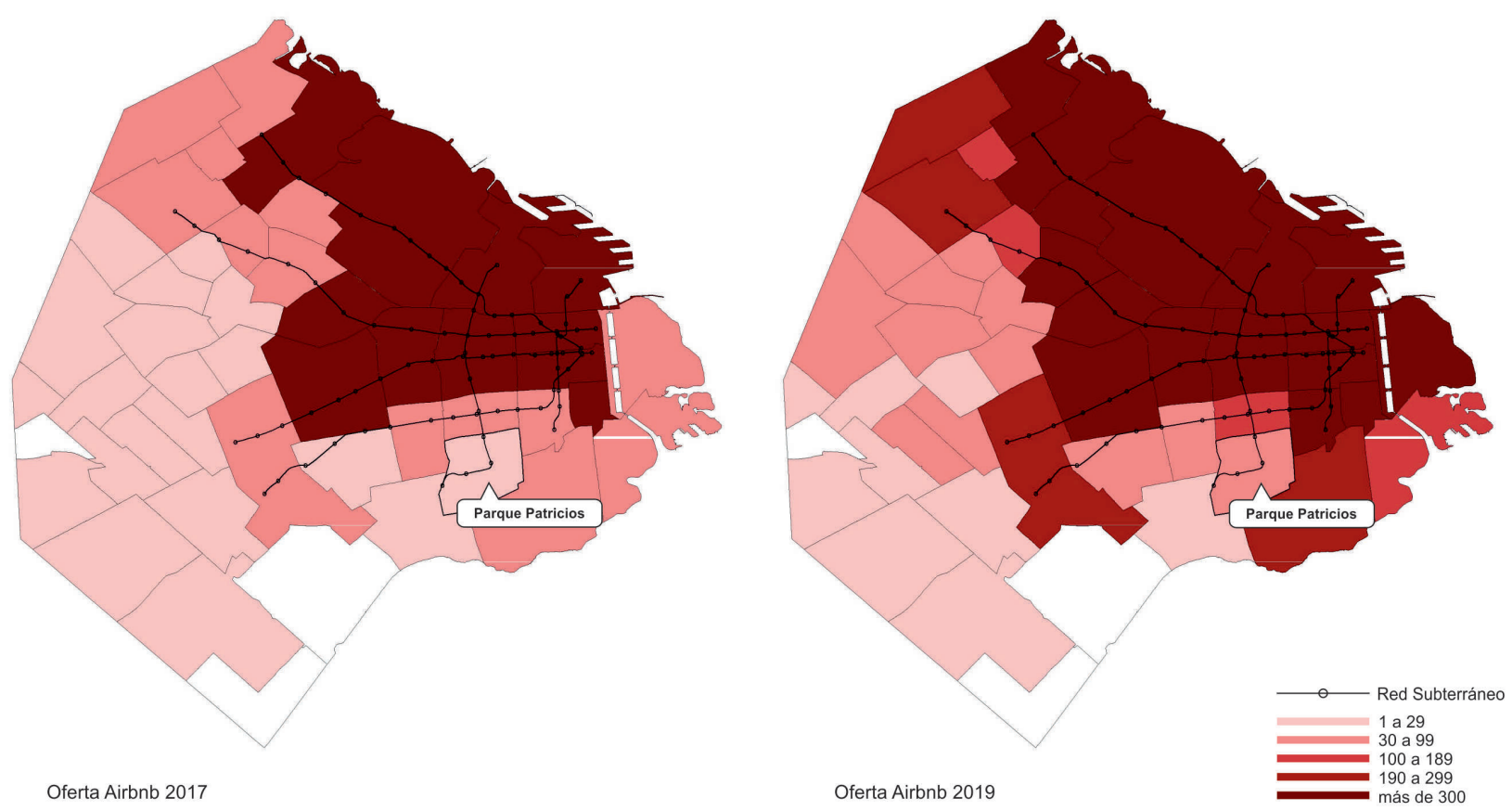

Fuente: Elaboración propia a partir de los datos obtenidos en la plataforma Airbnb. 
Figura 4. Oferta de Airbnb en Parque Patricios. 12 de octubre de 2017.

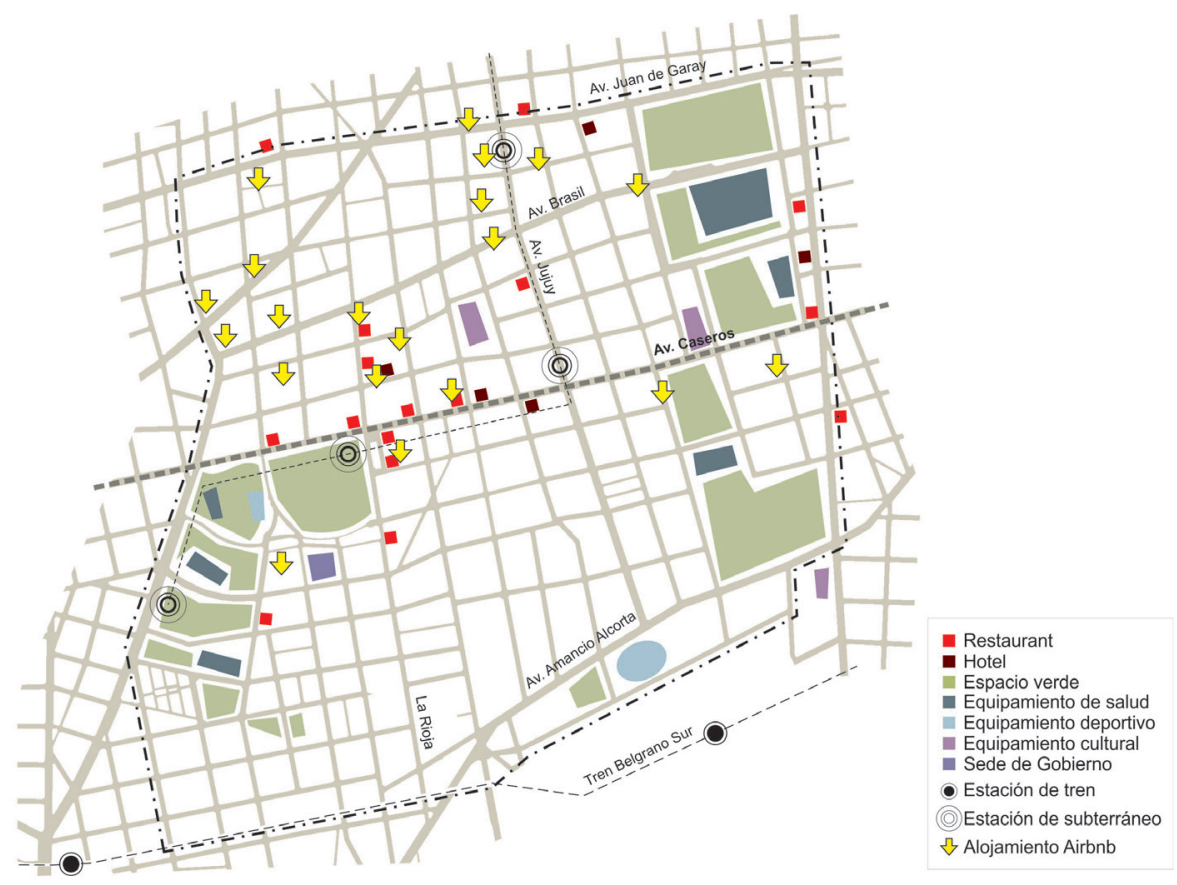

Fuente: Elaboración propia a partir de los datos obtenidos en la plataforma Airbnb.

Figura 5. Oferta de Airbnb en Parque Patricios. 04 de Mayo de 2019.

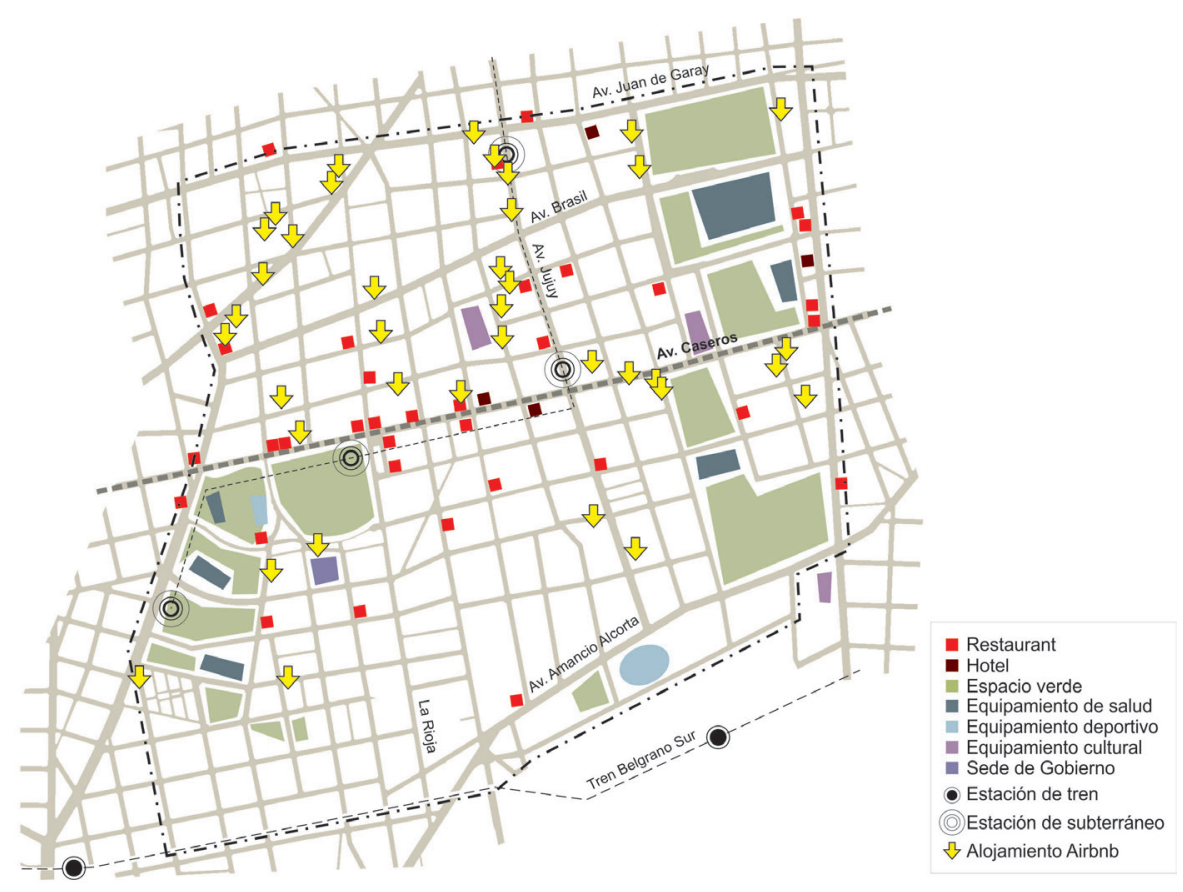

Fuente: Elaboración propia a partir de los datos obtenidos en la plataforma Airbnb.

Como fue señalado en el apartado metodológico, la información obtenida a través de los comentarios de anfitriones y visitantes, se clasificó en cuatro categorías: a) cualidades del barrio, b) accesibilidad y oferta de transporte público, c) oferta comercial barrial, y d) atractivos turísticos. Los comentarios sobre las cualidades del barrio destacan la tranquilidad y seguridad de la zona, o barrio, como sus características principales. La identificación del sector como una zona residencial, de casas bajas, típico de la ciudad de Buenos Aires, se muestran también como un atractivo. Al respecto, algunos comentarios lo mencionaban como un área no turística, lo que permitía una buena oportunidad para ver la vida real de los porteños (Airbnb, 2017). 
En cuanto a la accesibilidad y oferta de transporte, la cercanía al metro y la presencia de múltiples líneas de colectivo (a veces referidos como amplia oferta de transporte público) son las características más destacadas dentro de los cuatro grupos de comentarios 4 . Asociadas a estas observaciones, se acentúa la accesibilidad del barrio a puntos turísticos consolidados de la ciudad, especialmente el centro histórico o el microcentro, y los barrios de San Telmo y La Boca.

Respecto de la oferta comercial barrial, los visitantes mencionan como una virtud, la variedad de locales comerciales que posee el sector. A esa oferta, compuesta por comercios y servicios tradicionales (panadería, verdulería, farmacia, supermercado, bancos, etc.) se suma también la de locales gastronómicos, que parece replicar un modelo presente en otros puntos de la ciudad: cervecerías, bodegones, comida étnica, de autor, etc.; capitalizando la estética barrial como un atractivo. Estos locales, sin embargo, no están orientados especialmente al público turista, sino que parecen vincularse con una demanda emergente a partir de los nuevos trabajadores de las empresas TICs y Jefatura de Gobierno que llegan al barrio. En relación a este aspecto, el mapeo desarrollado a través de la web TripAdvisor (Figuras 4 y 5) permitió constatar que la oferta de restaurantes se duplicó en un lapso de año y medio (15 locales en 2017 contra 33 en 2019) y que su concentración se alinea a la locación de los alojamientos de Airbnb.

La categoría atractivos turísticos es la que menos comentarios concentra, tanto por oferentes como por huéspedes. Este es un punto a destacar, ya que evidencia que no existe impulso público ni privado por señalar atractivos dentro del barrio, a diferencia de los que sucede en los sitios turísticos consolidados de la ciudad, principalmente localizados en la zona centro y norte ${ }^{5}$. Los puntos de interés o atractivos que se mencionan son: el Club Atlético Huracán, la Casa de Gobierno, espacios verdes, ciertas actividades culturales y, aunque intangible, la historia del barrio. La mención de Parque Patricios como parte del DT, identidad principalmente promovida desde el gobierno local, tampoco es muy frecuente entre los comentarios. En relación a esta categoría, el relevamiento permitió identificar algunos servicios de excursiones y la inclusión del barrio en tours privados, como el City Tour Deportivo, que ofrece un recorrido por diferentes estadios de fútbol de la ciudad, donde se incluye el Estadio de Huracán ${ }^{6}$ o El otro Sur que promueve "un viaje al pasado y presente de los viejos barrios populares, en el que la inmigración, el ferrocarril y el tranvía, los obreros, el tango, el fútbol y la literatura son protagonistas"7. Sin embargo, ni el barrio como conjunto ni sus atractivos específicos, son promocionados desde el Ente de Turismo del Gobierno de la Ciudad.

Figura 6. Datos recogidos a través de la plataforma Airbnb.

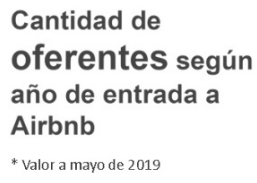

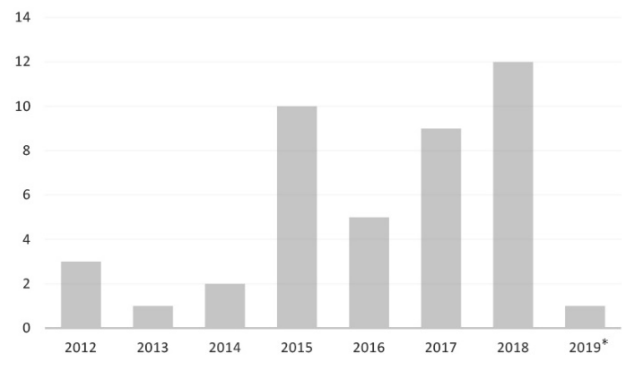

Descripción del barrio

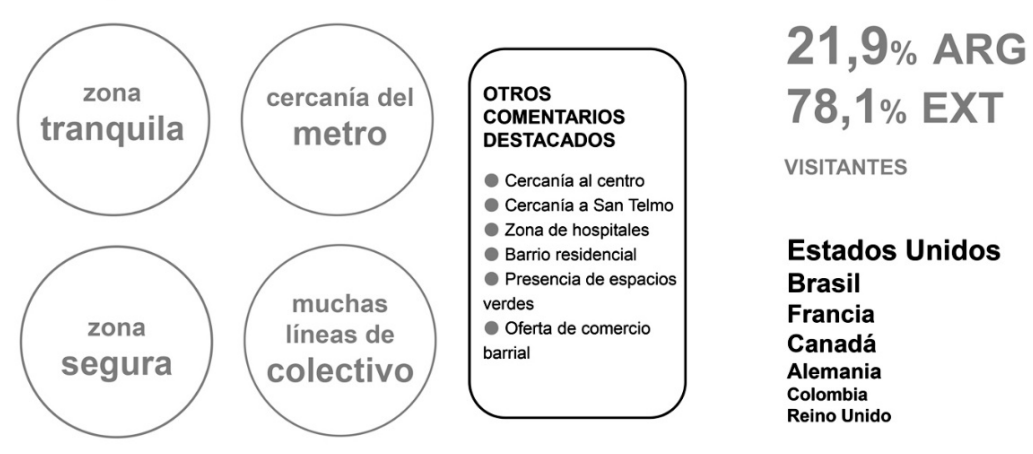

Fuente: Elaboración propia a partir de los datos obtenidos en la plataforma Airbnb.

$4 \quad$ Acerca de las condiciones de accesibilidad como atributo central del barrio, pueden consultarse Blanco et al., 2015; Apaolaza, et al. 2016 y Lerena, et al., 2016.

$5 \quad$ Ver Sitio Oficial de Turismo del Gobierno de la Ciudad: https://turismo.buenosaires.gob.ar/es

6 Buenos Aires, City Tour Deportivo: http://www.argentinainside.com/sp/asp/buenos_aires

7 Tours en Buenos Aires: El otro Sur: http://www.eternautas.tur.ar/tours-privados.php 
En relación al perfil de los huéspedes o visitantes que realizaron comentarios en cada una de las viviendas ofertadas ( 73 en total), la Figura 6 ilustra que un poco más del 20\% corresponde a argentinos (16), mientras que el resto (57) corresponde a visitantes extranjeros. Si se desglosan los visitantes por país, el predominio corresponde a Estados Unidos (10 visitantes), seguido por Brasil (6 visitantes).

En cuanto al valor de los alquileres ofrecidos por Airbnb en Parque Patricios estos oscilaban, para el último relevamiento (mayo de 2019), entre los 10 y los 34 dólares por persona la noche. Al comparar estos valores con los alquileres de residencia fija para el promedio de la Ciudad ${ }^{8}$, se observa que para abril del mismo año, el precio promedio de alquiler mensual de un departamento de tres ambientes de $70 \mathrm{~m}^{2}$ fue de 470 dólares (GCBA-DGEyC), a razón de 15,60 dólares por día 9 . Si consideramos el promedio de las ofertas de Airbnb para el barrio (22 dólares por noche) puede observarse cómo, en términos de rentabilidad, ofrecer la propiedad como alojamiento temporal puede resultar un mejor negocio que alquilarla de manera permanente. De continuar creciendo esta dinámica, considerando la tendencia a la inquilinización de la Ciudad (ACIJ, 2016; CEyS, 2016) y los porcentajes para Parque Patricios en particular, Airbnb podría entrar en tensión con el sistema local de alquileres, constriñendo el acceso a la vivienda para residentes locales.

\section{Hallazgos y conclusiones}

Este trabajo se propuso desarrollar una primera exploración a la emergencia de nuevas actividades económicas en el barrio de Parque Patricios, fuera de aquellas impulsadas directamente por el gobierno local. Entre los principales hallazgos, se destaca la estrategia metodológica desplegada: para el caso de la CABA, no existen trabajos que analicen las ofertas de alquileres temporales vía Airbnb como indicador de renovación urbana o de valorización del espacio en general. Se trata de una primera aproximación, exploratoria, quedando pendiente para próximos trabajos un análisis pormenorizado de diversas variables que no han sido abordadas en profundidad, como la evolución de la plataforma y la variación de precios entre diferentes barrios.

En el marco de una fuerte intervención política para la renovación de la zona sur de la CABA, se observa en Parque Patricios el surgimiento de servicios de alojamiento temporal vía Airbnb, habitualmente vinculados con la actividad turística. A diferencia de otras áreas de la ciudad, como San Telmo o La Boca, la renovación urbana de Parque Patricios no es impulsada activamente desde el Estado como destino turístico, sino como un polo para el desarrollo de industrias creativas. Sin embargo, los comentarios relevados a través de la plataforma en cuestión revelan que un número creciente de turistas elige hospedarse en Parque Patricios, algo que hasta hace poco más de siete años no sucedía. Esto parece denotar que, al contrario de lo que sucede en otros barrios, no es la actividad turística quién impulsa los procesos de renovación urbana, sino que más bien, la actividad es resultado de nuevos procesos de valorización, que generan condiciones para la emergencia del turismo.

Existe una fuerte correlación entre el mejoramiento de la infraestructura y la puesta en valor de la zona a partir de la creación del DT y el surgimiento de nuevos servicios de alojamiento temporal desde el sector privado. Paulatinamente, el barrio de Parque Patricios se va incorporando en el mapa de la Buenos Aires deseada por diversos actores: los inversores inmobiliarios en nuevas oficinas TICs, los mismos empresarios y trabajadores de estas empresas, e incluso visitantes o turistas, quienes descubren un nuevo barrio porteño (Lerena y Rodríguez, 2017).

El trabajo desarrollado permite señalar que la presencia de alojamientos temporales, a través de Airbnb, es una actividad incipiente, espontánea y de un crecimiento sostenido, donde la cantidad de hospedajes ofrecidos se ha cuadruplicado entre 2012 y 2019. A través de los comentarios de anfitriones y visitantes (fundamentalmente extranjeros), puede concluirse que los elementos centrales que explican el surgimiento de esta nueva oferta en Parque Patricios son la proximidad y buena accesibilidad a los centros turísticos del centro-sur de la ciudad (Centro, San Telmo y La Boca), así como también las cualidades barriales de tranquilidad, seguridad o genuinamente porteño a explorar. Los visitantes parecen otorgar especial valor a los espacios de la vida cotidiana, expresados en las menciones acerca de la oferta comercial barrial como supermercados, verdulerías, farmacias, etc., aunque no hay referencias acerca de conveniencia económica.

También puede observarse que en Parque Patricios, fuera de la oferta de alojamiento temporal, no hay actividades específicamente orientadas al turismo, sino que los visitantes se encuentran siendo parte de dinámicas del barrio, pero aprovechando la accesibilidad a sitios turísticos de la ciudad. Asimismo, cabe señalar que la posibilidad misma de ser parte de la red Airbnb da cuenta de estándares mínimos de calidad que son alcanzados en estas ofertas, lo que habla de un cierto grado de competitividad de las unidades

$8 \quad$ No se dispone de este dato para el barrio de Parque Patricios.

9 Para analizar este dato, es necesario tener en cuenta que, al tratarse de un promedio de la Ciudad, este valor muy posiblemente sea aún más bajo en Parque Patricios. 
residenciales ofrecidas.

Hasta el momento, las ofertas de alojamiento temporal son escasas en comparación con destinos turísticos consolidados de la ciudad (Figura 6); lo mismo acontece con otras ofertas de servicios que pueden asociarse a la actividad turística, como el sector gastronómico -aunque se encuentra en crecimiento, como puede verse a través de TripAdvisor-. Sin embargo, es posible afirmar que, aunque incipiente, el surgimiento de servicios de alojamiento temporal en Parque Patricios es un indicador más de las transformaciones que tienen lugar en este barrio, desde su consideración pública como área a renovar.

En un barrio donde el 40\% de sus residentes son inquilinos (INDEC, 2010), y con precios de alquiler más rentables para el alojamiento temporal que para alquileres de vivienda, cabe preguntarse sobre la tensión que pueda generarse entre la rentabilidad de los propietarios Airbnb y la sostenida demanda de los residentes locales. Esto es especialmente sensible, por cuanto el acceso a la vivienda en la Ciudad Autónoma de Buenos Aires se encuentra totalmente orientado por el mercado, y porque en las políticas de renovación urbana desplegadas no se prevén mecanismos para compensar los procesos de valorización excluyente asociados.

\section{Referencias bibliográficas}

Asociación Civil por la Igualdad y la Justicia (ACIJ) (2016). Buscar un techo. La problemática de los inquilinos y los alquileres en la Ciudad Autónoma de Buenos Aires. Colección Es Nuestra La Ciudad, 2. Recuperado de: https://esnuestralaciudad.org/wp-content/uploads/2016/07/Coleccion-PP-ACIJ_ Cuadernillo-n2_Alquileres_julio-2016_impresion.pdf

Almirón, A.; Bertoncello, R.; Kuper, D. y Ramírez, L. (2008). El turismo como impulsor del desarrollo en Argentina. Una revisión de los estudios sobre la temática. Aportes y Transferencias, 12(1), 57-86.

Apaolaza, R.; Blanco, J.; Lerena, N.; Lopez Morales, E.; Lukas, M. y Rivero, M. (2016). Transporte, desigualdad social y capital espacial: análisis comparativo entre Buenos Aires y Santiago de Chile. Íconos, 56, 19-41. Recuperado de: http://revistas.flacsoandes.edu.ec/iconos/article/view/2148/1484

Baum, T. (1994). The development and the implementation of national tourism policies. Tourism Management, 15(3), 185-192.

Bertoncello, R. y Troncoso, C. (2014). La ciudad como objeto de deseo turístico. Renovación urbana, cultura y turismo en Buenos aires y Salta (Argentina). Gran Tour, 9, 4-26.

Blanco, J., Apaolaza, R. y Lerena, N. (2015). Gentrificación, movilidad y capital espacial: una exploración del barrio de Parque Patricios, Buenos Aires. Conflictos urbanos y territoriales: ¿desafiando la cohesión social? Conferencia Anual COES 2015, Santiago, Chile.

Borja, J. y Castells, M. (1997). Local y global. Madrid: Taurus

Borja, J. (2003). La ciudad conquistada. Madrid: Alianza

Britton, S. (1991). Tourism, capital, and place: towards a critical geography of tourism. Environment and Planning: D Society and Space, 9(4), 461- 478.

Consejo Económico y Social de la ciudad de Buenos Aires CEyS (2016). La ciudad de Buenos Aires inquilinizada. Recuperado de: http://www.bdigital.cesba.gob.ar/handle/123456789/90

Ciccolella, P. y Mignaqui, I. (2009). Globalización y transformaciones de la centralidad histórica en Buenos Aires. Centro-h, 3, 91-101. Recuperado de: http://www.redalyc.org/articulo.oa?id=115112536008

Comisión Europea. (2016). Una Agenda Europea para la economía colaborativa. Comunicación de la Comisión al Parlamento Europeo, al Consejo, al Comité Económico y Social Europeo y al Comité de las Regiones. Bruselas.

Delgadillo, V. (2015). Patrimonio urbano, turismo y gentrificación. En: V. Delgadillo, I. Díaz y L. Salinas, Perspectivas del Estudio de la Gentrificación en México y América Latina (pp. 113-132). Coyoacán: Instituto de Geografía, UNAM.

De Mattos, C. A. (2004). De la planificación a la governance: implicancias para la gestión territorial y urbana. Revista Paranaense de Desenvolvimiento, 07, 9-23

Di Virgilio, M. y Guevara, T. (2014). Gentrificación liderada por el Estado y empresarialismo urbano en la Ciudad Autónoma de Buenos Aires. Estudios Sociales Contemporáneos, 11, 12-23. 
Fernández Güell, J. M. (1997). Planificación estratégica de ciudades. Barcelona: Gustavo Gilli.

Goicoechea, M. E. (2016). Distritos creativos en el Sur de la Ciudad de Buenos Aires (2008-2015). Renovación urbana y nuevas lógicas de segregación. Tesis doctoral. Universidad de Buenos Aires, Facultad de Ciencias Sociales.

Goicoechea, M. E. (2017). Renovación urbana en el sur porteño y el “éxito” del Distrito Tecnológico. Algunas claves para comprender el dinamismo inmobiliario. Quid 16: Revista del área de Estudios Urbanos, 6, 30-61.

Goicochea, M. E. (2018). Distritos Creativos en el sur de la Ciudad de Buenos Aires (2008-2015). Renovación urbana y nuevas lógicas de segregación. Quid 16: Revista del área Estudios Urbanos, 9, 224-227.

Gómez Schettini, M. (2009_a). Del atractivo turístico auténtico a la construcción de autenticidad. Turismo y renovación urbana en el barrio de La Boca de la ciudad de Buenos Aires. En: R. Bertoncello (Comp.), Turismo y geografía: Lugares y patrimonio natural-cultural de la Argentina (pp. 111-133). Buenos Aires, Argentina: Ediciones Ciccus.

Gómez Schettini, M. (2009_b). El turismo y la cultura en la renovación de Buenos Aires. Entre la necesidad y la especulación. En: L. Maronesse (Resp.), Turismo Cultural II. Temas de Patrimonio Cultural. Buenos Aires, Argentina.

Green, R. (2005). Pensar, dibujar, matar la ciudad: orden, planificación y competitividad en el urbanismo moderno. Revista EURE, 94, 77-95.

Gutiérrez, J., García-Palomares, J., Romanillos, G. \& Salas-Olmedo, M. (2017). The eruption of Airbnb on tourist cities: Comparing spatial patterns of hotels and peer-to-peer accommodation in Barcelona. Tourism Management, 62, 278-291.

Guttentag, J. (2015). Airbnb: disruptive innovation and the rise of an informal tourism accommodation sector. Current Issues in Tourism, 18(12), 1192-1217.

Harvey, D. (1989). From managerialism to entrepreneurialism: The transformation in urban governance in late capitalism. Geografiska Annaler, 71(1), 3-17.

Harvey, D. (2013). Ciudades Rebeldes. Del derecho de la ciudad a la revolución urbana. Madrid: Akal.

Harvey, D. (2016). Diecisiete contradicciones y el fin del capitalismo. Madrid: Traficantes de sueños.

Herzer, H. (2008). Con el corazón mirando al Sur. Transformaciones en el Sur de la Ciudad de Buenos Aires. Buenos Aires: Espacio Editorial.

Hiernaux, D. y González, C. (2014). Turismo y gentrificación: pistas sobre una articulación. Revista de Geografía Norte Grande, 58, 55-70.

INDEC. (2010). Censo Nacional de Población, Hogares y Viviendas. Recuperado de: https://www. indec.gob.ar/indec/web/Nivel4-Tema-2-41-135

Janoschka, M., Sequera J., y Salinas, L. (2014). Gentrificación en España y América Latina: Un diálogo crítico. Revista de geografía Norte Grande, 58, 7-40.

Lerena, N. (2019). Diez años del Distrito Tecnológico: políticas de desarrollo urbano y valorización inmobiliaria en el sur de la Ciudad de Buenos Aires. Quid 16: Revista del área Estudios Urbanos, 11, 201-221.

Lerena, N. (2016). Planificación urbana estratégica, transformaciones territoriales y derecho a la ciudad: el caso del Distrito Tecnológico de Parque Patricios (CABA). XXX Jornadas de investigación y XII Encuentro Regional SI+FADU. Ciudad de Buenos Aires, Argentina.

Lerena, N. y Rodríguez, L. (2017) “Renovación urbana en Parque Patricios: ¿hacia la configuración de un nuevo destino turístico en el Distrito Tecnológico?" en III Jornadas Internacionales de Antropología del Conflicto Urbano. Buenos Aires, 13-15 diciembre.

Lerena, N.; Tillman, R. y Apaolaza, R. (2016). Sobre subterráneos, bicisendas y ciudades para pocos. La doble función del transporte en los procesos de gentrificación en Parque Patricios, Buenos Aires. Congreso Internacional Contested Cities, Eje 4: Gentrificación. Serie (IV-4B). Disponible en: http://contested-cities. net/working-papers/category/2016/

Porter, M. (1995). The competitive advantage of the inner city. Harvard Bussiness Review. 55-71. Disponible en: https://hbr.org/1995/05/the-competitive-advantage-of-the-inner-city\#

Porter, M. (1998). Clusters and the new economic competition. Harvard Business Review. Disponible en: https://hbr.org/1998/11/clusters-and-the-new-economics-of-competition 
Rodríguez, M., Arqueros Mejica, S., Rodríguez, F., Gómez Schettini, M. y Zapata, M. (2011). La política urbana "pro": continuidades y cambios en contextos de renovación en la Ciudad de Buenos Aires. Cuaderno Urbano: espacio, cultura y sociedad, 11(11), 101-121.

Sargatal, M. (2009). El barrio del Raval de Barcelona (1999-2008). Transformaciones urbanas y nuevos enfoques metodológicos para el estudio del centro histórico. Biblio $3 W$, Revista Bibliográfica de Geografía y Ciencias Sociales, 14 (824). s/p.

Smith, N. (1979). Toward a theory of gentrification: a back to the city movement by capital, not people. Journal of the American Planning Association, 45, 538-548.

Socoloff, I., Colombo, A., Kitay, I., Maltz, H., Rullansky, I., Seia, G., Stiberman, L. y Urdampilleta, M. (2012). Gobernar Buenos Aires. Un estudio sobre las racionalidades políticas en torno al desarrollo local a partir del caso del Distrito Tecnológico de Parque Patricios (2008-2012). VII Jornadas de Sociología. La Plata, Buenos Aires, Argentina.

Thomasz, A. (2016). Los nuevos distritos creativos de la Ciudad de Buenos Aires: la conversión del barrio de La Boca en el "Distrito de las Artes". Revista EURE, 42(126), 123-144. Disponible en: https:/| dx.doi.org/10.4067/S0250-71612016000200007

Vecslir, L. y Rodríguez, L. (2018). Centros Comerciales a Cielo Abierto como política de renovación de las centralidades tradicionales en el conurbano bonaerense. Territorios, 38, 15-40.

Yrigoy, I. (2016). The impact of AirBnb in the urban arena: towards a turism-led gentrification? The case-study of Palma old quarter (Mallorca, Spain). Congreso Internacional Contested Cities, Madrid: UAM.

\subsection{Fuentes electrónicas}

Airbnb. (2017). Sobre nosotros. Disponible en: https://www.airbnb.com.ar/about/about-us (Consulta: 13/10/2017)

Clarín. (2016). Buenos Aires, la preferida de Sudamérica. Disponible en: http://www.clarin.com/ ciudades/ciudad_de_buenos_aires-ranking-tripadvisor-sudamerica_0_By1e2HR_Dml.html (Consulta: 26 de Enero de 2017) 